\title{
A Clinical Observation of Intravenous Thrombolysis in Acute Ischemic Stroke with Minor Trauma
}

\section{Yansen Cui Liquan Wang}

Department of Emergency, Linyi People's Hospital, Linyi, 27600I, People's Republic of China
Correspondence: Liquan Wang Department of Emergency, Linyi People's Hospital, Wohushan Street, Lanshan District, Linyi, 276003, Shandong, People's Republic of China

Tel +865398129120

Email wanglqdoc52@I63.com
Objective: This study aimed to observe the curative effect of intravenous thrombolysis with alteplase in patients with acute ischemic stroke and minor trauma.

Methods: The data of 25 patients with minor trauma who were treated in our department from January to December 2018 were analyzed retrospectively (observation group), and during the same period, 25 thrombolytic patients were enrolled as the control group. Brain computed tomography (CT) was performed before and at three days after thrombolysis. NIHSS scores were recorded before thrombolysis and at one, three, and seven days after thrombolysis. Additionally, bleeding rate after thrombolysis and 90-day mRS scores were recorded.

Results: The differences in 1-day, 3-day, and 7-day NIHSS scores and 90-day mRS scores between the two groups were not statistically significant $(\mathrm{P}>0.05)$. The bleeding rate was higher in the observation group than in the control group, and this was statistically significant $(\mathrm{P}<0.05)$.

Conclusion: Intravenous thrombolysis with alteplase has a good curative effect in acute ischemic stroke patients with minor trauma. The bleeding rate was significantly higher than in the control group, but there was no significant difference in the long-term curative effect. Keywords: alteplase, acute ischemic stroke, the curative effect and safety, intravenous thrombolysis

\section{Introduction}

Stroke is the most common cerebrovascular disease, with a high disability rate, high recurrence rate, and high mortality. ${ }^{1-3}$ According to the World Health Organization (WHO), the increasing trend of incidence of strokes in China will continue for 30 years. The treatment cost for it is high. ${ }^{4,5}$ From 1990 to 2013, the prevalence of ischemic strokes increased by $28.8 \%$ in China, which led to heavy burdens to society and families. ${ }^{6,7}$ Because of the rising trend of new cases every year, the disease has become the primary cause of death in China. In stroke cases, $78-80 \%$ are ischemic strokes. ${ }^{8}$

Alteplase (rt-PA) is a recombinant tissue plasminogen activator. By binding with fibrin, it activates plasminogen to convert into fibrinolysin, which dissolves thrombus. ${ }^{9}$ At present, the first choice for the treatment of acute ischemic stroke (AIS) in all countries is to give the thrombolytic therapy of alteplase within $4 \frac{1}{2}$ hours. This has been confirmed by many units and recommended by multinational guidelines. ${ }^{10-14}$ At present, both American and Chinese guidelines list severe brain 
injury as the contraindication of intravenous thrombolysis ${ }^{15}$ and injuries in other regions of the body without brain injury as a relative contraindication but do not give clear treatment opinion for patients with mild craniocerebral injury or mild trauma in other parts. Since many patients with AIS often demonstrate traumatic behaviors such as falling from beds and falling down generally, these patients often have head, face, limb, and trunk injuries. The specific manifestations of these include bruises, congestion, cyanosis, and swelling of the injured area. From January to December 2018, in order to observe the effect and safety of intravenous thrombolysis in these patients, we analyzed 25 patients with AIS and mild trauma who underwent intravenous thrombolysis in our neurology department. Details are reported as follows.

\section{Materials and Methods}

\section{General Information}

In this study, 819 patients with AIS who underwent intravenous thrombolysis with alteplase from January 2018 to December 2018 were analyzed retrospectively, and 25 patients with AIS and mild trauma were screened as the observation group. During the same period, 25 thrombolytic patients were enrolled as the control group. The National Institutes of Health Stroke Scale (NIHSS) scores of all patients before thrombolysis ranged within 4-25 points. None of the selected cases were treated with endovascular thrombectomy. The patients' ages, genders, and histories of hypertension, diabetes, heart issues, hyperlipidemia, smoking, and antiplatelet drugs, as well as NIHSS scores before thrombolysis for each group, were compared.

\section{Inclusion Criteria}

(1) All the patients included met the diagnostic criteria of AIS. ${ }^{16-18}$ According to the guidelines, the indications of intravenous thrombolysis were met, and the contraindications of intravenous thrombolysis were excluded.

(2) All patients in the observation group had different degrees of trauma in the head, face, limbs, trunk, and other parts. The contraindications of intravenous thrombolysis, such as intracranial hemorrhage, cerebral contusion and laceration, visceral injury, fracture of limbs, and other parts, were excluded.

\section{Methods}

Before thrombolysis, the patients in both groups were excluded from intracranial hemorrhage or early cerebral infarction (disappearance of sulcus). Patients with lacunar infarction or old cerebral infarction without obvious symptoms and signs could also be included. No contraindication of thrombolysis was found in routine blood coagulation tests. All patients were given $0.9 \mathrm{mg} / \mathrm{kg}$ (maximum $90 \mathrm{mg}$ ) of articipase rt-PA (Berlin Ingelheim company, Germany) for thrombolysis: $10 \%$ of the total dose was given intravenously for one minute, and the remaining dose was pumped in within one hour. The door-to-needle time (DNT) was approximately 40 minutes. CT scans were performed at 24 hours after thrombolysis. If intracranial hemorrhage was excluded, $200 \mathrm{mg}$ of aspirin (qd) was given. Within 72 hours, brain magnetic resonance angiography (MRA), magnetic resonance imaging (MRI), and susceptibility weighted imaging (SWI) were performed to observe the infarct area, intracranial vessels, and the presence of post-thrombolysis hemorrhage. Diffusionweighted imaging (DWI) was performed to diagnose super-early cerebral ischemia. MRI + MRA + DWI + SWI was performed to every patient's MRI examination. The NIHSS scores before thrombolysis and at 24 hours, three days, and seven days after thrombolysis, 72-hour hemorrhage rate, and 90-day mRS scores were calculated. Clinical efficacy was assessed based on NIHSS score, safety was assessed based on cerebral hemorrhage transformation, and the prognosis was evaluated based on mRS score: $0-2$ points $=$ good prognosis and non-disability; 3-6 points $=$ poor prognosis and disability. ${ }^{19}$ The postthrombolysis NIHSS scores of the two groups were compared to evaluate the clinical efficacy of intravenous thrombolysis. The incidence of cerebral hemorrhages after thrombolysis was counted to assess the safety of the treatment. The 90-day mRS scores of the two groups were compared to evaluate the prognosis.

\section{Statistical Analysis of Data}

Data were statistically analyzed using statistical software SPSS22.0. The measurement data of the two groups were compared using a $t$-test. Count data were expressed as the number of cases or as a percentage and were compared using an $X^{2}$ test. $\mathrm{P}<0.05$ was considered statistically significant.

\section{Results General Data}

A total of 25 patients with AIS and minor trauma were included as the observation group. Among these, 18 patients were male, and 7 patients were female. The patients were 
Table I Comparison of General Conditions Between the Two Groups

\begin{tabular}{|l|c|c|c|}
\hline Item & $\begin{array}{c}\text { Observation } \\
\text { Group (n=25) }\end{array}$ & $\begin{array}{c}\text { Control } \\
\text { Group (n=25) }\end{array}$ & $\mathbf{P}$ \\
\hline Age & $64.2 \pm 10.6$ & $65.7 \pm 9.7$ & 0.3659 \\
\hline Male & 18 & 17 & 9.5238 \\
\hline Hypertension & 12 & 13 & 0.08 \\
\hline Diabetes & 8 & 9 & 0.0891 \\
\hline Heart disease & 6 & 5 & 0.1165 \\
\hline Smoking & 13 & 14 & 0.7773 \\
\hline Drinking & 10 & 9 & 0.7773 \\
\hline $\begin{array}{l}\text { NIHSS Score } \\
\text { Before } \\
\text { thrombolysis }\end{array}$ & $11.01 \pm 4.57$ & $10.75 \pm 5.51$ & 0.3659 \\
\hline
\end{tabular}

aged between $37-84$ years, and the average age was $64.2 \pm$ 10.6 years. During the same period, 25 thrombolytic patients were enrolled as the control group. Among these, 17 patients were male, and 8 patients were female. The patients were aged between $37-85$ years, and the average age was $65.7 \pm$ 9.6 years. The differences in age, gender, history of hypertension, diabetes, heart disease, hyperlipidemia, smoking, antiplatelet drugs, and NIHSS score before thrombolysis between the two groups were not statistically significant $(P>0.05)$. Hence, the two groups were comparable (Table 1).

\section{NIHSS Score and mRS Score of the Two Groups After Treatment}

The NIHSS scores of the two groups before treatment, and at one, three and seven days after treatment were compared. The results revealed that the neurological functions of both groups effectively improved after treatment. No significant differences were found in the NIHSS scores at one, three and seven days after treatment between the two groups $(P>0.05)$, and there was no significant difference in the 90-day $\mathrm{mRS}$ scores $(P>0.05$, see Table 2 for details). Besides, we found that the NIHSS score of patients in both groups decreased with time.

\section{Bleeding After Treatment in Two Groups}

Safety evaluation was conducted through statistical analysis of the occurrence of gingival bleeding, subcutaneous bleeding, and cerebral hemorrhage in the two groups. Of the 25 patients in observation group, 5 patients presented with gingival bleeding, 7 patients with subcutaneous bleeding, 6 patients with cerebral hemorrhage; while in the control group, 5 patients with gingival bleeding, none with subcutaneous bleeding, 3 patients with cerebral hemorrhage. Statistical results revealed that the difference in the incidence of gingival bleeding after thrombolytic therapy between the two groups was not statistically significant $(\mathrm{P}>0.05)$. However, the differences in the incidence of subcutaneous bleeding and cerebral hemorrhage between the two groups were statistically significant $(\mathrm{P}<0.05$, see Table 3 for details).

\section{Discussion}

Ischemic stroke consists of local cerebral ischemia and necrosis, which is caused by a local cerebral blood flow disorder. The shorter the time interval from the onset of a stroke to the start of thrombolysis, the greater the health benefit to patients will be. ${ }^{20}$ Therefore, the key to the treatment of AIS is to open the occluded vessels as soon as possible, restore the blood flow, and save the ischemic penumbra. $^{21}$ Studies have revealed that alteplase can dissolve thrombus and therefore achieve this most effective treatment at the acute stage. ${ }^{22-24}$ However, the risk of hemorrhagic transformation of thrombolysis with alteplase greatly limits its clinical application. Because of the acute onset of AIS, external traumas could be made from a brief loss of consciousness or sudden weakness in the limbs occurred to patients. The patients may also be prone to falling out of bed and incur trauma when they suffer from strokes in bed. Severe trauma can cause craniocerebral injury and intracranial hemorrhage, while mild trauma can cause skin redness, pain, swelling, cyanosis, tenderness, and bleeding. These mild traumas are mainly caused

Table 2 NIHSS Score and mRS Score of Patients in the Two Groups After Treatment

\begin{tabular}{|l|c|c|c|}
\hline Item & IdNIHSS Score & 3dNIHSS Score & 7dNIHSS Score \\
\hline Observation group $(n=25)$ & $5.24 \pm 1.3626$ & $4.12 \pm 1.2689$ & $2.56 \pm 1.4457$ \\
Control group $(n=25)$ & $5.16 \pm 1.65$ & $4.0 \pm 1.22$ & $2.88 \pm 1.0536$ \\
$P$ & 0.7747 & 0.6639 & 0.0727 \\
\hline
\end{tabular}


Table 3 Bleeding Situation of Patients in the Two Groups After Treatment

\begin{tabular}{|l|c|c|c|}
\hline Item & Gingival Bleeding Rate & Subcutaneous Bleeding & Cerebral Hemorrhage \\
\hline Observation group $(n=25)$ & 5 & 7 & 6 \\
Control group $(n=25)$ & 5 & 0 & 3 \\
P & & & \\
\hline
\end{tabular}

by the damage to the soft tissue cells of superficial muscle and the rupture of microvessels. In this case, the application of alteplase can cause hematoma aggravation and local bleeding in the injured area. Different degrees of intracranial hemorrhage occurred in six patients, with the incidence being significantly higher than in the control group. However, the bleeding in these patients was not from the infarct area but was from a small amount of subdural or extradural bleedings at the site of injury or the contraposition, and one patient had subarachnoid bleeding. All six patients had head and face trauma, as well as local hematoma formation. This suggests that the rate of bleeding transformation after thrombolysis is increased significantly in patients with hematoma after head and face trauma. The reason may be the early intracerebral hemorrhage without obvious hematoma after head and face injuries. Because brain CT cannot find such micro bleedings, ${ }^{25}$ and the use of thrombolytic drugs can increase bleeding, it may be that there is no bleeding in the early stage of craniocerebral injury. However, there are small injuries to the capillary walls, which can cause bleeding after thrombolysis. Therefore, for intravenous thrombolysis for AIS patients with trauma, if possible, brain MRI and SWI should be performed ${ }^{26}$ to observe whether there are micro-hemorrhages in the brain and assess the risk of thrombolysis. After thrombolysis, the patient's condition should be observed, and the imaging examination should be performed again. In particular, before starting antithrombotic or anticoagulant therapy, imaging examination must be carried out. However, the difference in the 90-day $\mathrm{mRS}$ score between the two groups was not statistically significant. The results revealed that these bleedings did not affect the prognosis of patients.

\section{Ethics Approval and Consent to Participate}

This study was conducted in accordance with the declaration of Helsinki. This study was conducted with approval from the Ethics Committee of Linyi People's Hospital.
A written informed consent was obtained from all participants.

\section{Author Contributions}

All authors made a significant contribution to the work reported, whether that is in the conception, study design, execution, acquisition of data, analysis and interpretation, or in all these areas; took part in drafting, revising or critically reviewing the article; gave final approval of the version to be published; have agreed on the journal to which the article has been submitted; and agree to be accountable for all aspects of the work.

\section{Disclosure}

The authors report no conflicts of interest in this work.

\section{References}

1. Tsivgoulis G, Safouris A, Alexandrov AV. Safety of intravenous thrombolysis for acute ischemic stroke in specific conditions. Expert Opin Drug Saf. 2015;14(6):845-864. doi:10.1517/ 14740338.2015.1032242

2. Cappellari M, Bovi P. Intravenous thrombolysis for stroke in patients taking non-VKA oral anticoagulants: an update. Thromb Haemost. 2015;114(2):440-444. doi:10.1160/TH14-11-0973

3. Huang X, Cheripelli BK, Lloyd SM, et al. Alteplase versus tenecteplase for thrombolysis after ischemic stroke (ATTEST): a phase 2, Randomized, Open-Label, Blinded Endpoint Study. Lancet Neurol. 2015;14(4):368-376. doi:10.1016/S1474-4422(15)70017-7

4. Bejot $\mathrm{Y}$, Catteau A, Caillier M, et al. Trends in incidence, risk factors, and survival in symptomatic lacunar stroke in Dijon, France, from 1989 to 2006: a Population-Based Study. Stroke. 2008;39 (7):1945-1951. doi:10.1161/STROKEAHA.107.510933

5. Lloyd-Jones D, Adams RJ, Brown TM, et al. American Heart Association statistics committee and stroke statistics subcommittee. heart disease and stroke statistics-2010 update: a report from the American Heart Association. Circulation. 2010;121(7):e46e215.

6. Fugate JE, Rabinstein AA. Update on intravenous recombine anttissue plasminogen activator for acute ischemic stroke. Mayo Clin Proc. 2014;89(7):960-972. doi:10.1016/j.mayocp.2014.03.001

7. Wechsler LR. The 4.5- hour time window for intravenous thrombolysis with intravenous tissue-type plasminogen activator is not firmly established. Stroke. 2014;45(3):914-915. doi:10.1161/ STROKEAHA.113.002701

8. Wang W, Jiang B, Sun H, et al.; NESS-China Investigators. Prevalence, incidence, and mortality of stroke in China: results from a nationwide population-based survey of 480687 adults. Circulation. 2017;135 (8):759-771. doi:10.1161/CIRCULATIONAHA.116.025250 
9. Leung TW, Wong KS. Thrombolysis with alteplase 3-4.5 h after acute ischemic stroke (SITS-ISTR): an Observational Study. Lancet. 2008;372(9646):1303-1309. doi:10.1016/S0140-6736(08)61339-2

10. Ahmed N, Wahlgren N, Grond M, et al.; SITS investigators. Implementation and outcome of thrombolysis with alteplase 3-4.5 $\mathrm{h}$ after an acute stroke: an updated analysis from SITS-ISTR. Lancet Neurol. 2010;9(9):866-874. doi:10.1016/S1474-4422(10)70165-4

11. Stroke Thrombolysis Trialists' Collaborative Group. Details of a prospective protocol for a collaborative meta-analysis of individual participant data from all randomized trials of intravenous rt-PA vs. control: statistical analysis plan for the stroke thrombolysis trialists' collaborative meta-analysis. Int $J$ Stroke. 2013;8(4):278-283. doi:10.1111/ijs. 12040

12. Lansberg MG, Bluhmki E, Thijs VN. Efficacy and safety of tissue plasminogen activator 3 to 4.5 hours after acute ischemic stroke: a meta analysis. Stroke. 2009;40(7):2438-2441. doi:10.1161/ STROKEAHA.109.552547

13. Kunisawa S, Kobayashi D, Lee J, et al. Factors associated with the administration of tissue plasminogen activator for acute ischemic stroke. Stroke Cerebrovasc Dis. 2014;23(4):724-731.

14. Ploneda Perilla AS, Schneck MJ. Unanswered questions in thrombolytic therapy for acute ischemic stroke. Neurol Clin. 2013;31 (3):677-704. doi:10.1016/j.ncl.2013.03.006

15. Powers WJ, Rabinstein AA, Ackerson T, et al; American Heart Association Stroke Council. 2018 Guidelines for the early management of patients with acute ischemic stroke. A guideline for healthcare professionals from the American Heart Association/American Stroke Association. Stroke. 2018;49(3):e46-e110. doi:10.1161/ STR.0000000000000158

16. Bracard S, Ducrocq X, Mas JL, et al.; THRACE investigators. Mechanical thrombectomy after intravenous alteplase versus alteplase alone after stroke (THRACE): a randomised controlled trial. Lancet Neurol. 2016;15(11):1138-1147. doi:10.1016/S1474-4422(16) 30177-6

17. Anderson CS, Woodward M, Arima H, et al.; ENCHANTED Investigators. Statistical analysis plan for evaluating low- vs. standard-dose alteplase in the Enhanced Control of Hypertension and Thrombolysis Stroke Study (ENCHANTED). Int J Stroke. 2015;10(8):1313-1315. doi:10.1111/ijs.12602
18. Campbell BC, Meretoja A, Donnan GA, Davis SM. Twenty-year history of the evolution of stroke thrombolysis with intravenous alteplase to reduce long-term disability. Stroke. 2015;46 (8):2341-2346. doi:10.1161/STROKEAHA.114.007564

19. Carvalho A, Cunha A, Gregório T, et al. Is the efficacy of endovascular treatment for acute ischemic stroke sex-related. Interv Neurol. 2018;7(1-2):42-47. doi:10.1159/000484098

20. Hacke W, Kaste M, Bluhmki E, et al.; ECASS Investigators. Thrombolysis with alteplase 3 to 4.5 hours after acute ischemic stroke. $N$ Engl J Med. 2008;359(13):1317-1329. doi:10.1056/ NEJMoa0804656

21. Romano JG, Smith EE, Liang L, et al. Outcomes in mild acute ischemic stroke treated with intravenous thrombolysis: a retrospective analysis of the get with the guidelines-stroke registry. JAMA Neurol. 2015;72(4):423-431. doi:10.1001/ jamaneurol.2014.4354

22. Wang DJ, Alger JR, Qiao JX, et al.; UCLA Stroke Investigators. The value of arterial spin-labeled perfusion imaging in acute ischemic stroke: comparison with dynamic susceptibility contrast-enhanced MRI. Stroke. 2012;43(4):1018-1024. doi:10.1161/ STROKEAHA.111.631929

23. Kim DH, Cha JK, Park HS, Choi JH, Kang MJ, Huh JT. Direct access to a hospital offering intravenous thrombolysis therapy improves functional outcome of acute ischemic stroke patients. $J$ Clin Neurosci. 2014;21(8):1428-1432. doi:10.1016/j.jocn.2014.03.014

24. Fisher M, Saver JL. Future directions of acute ischaemic stroke therapy. Lancet Neurol. 2015;14(7):758-767. doi:10.1016/S14744422(15)00054-X

25. Huang P, Chen CH, Lin WC, Lin RT, Khor GT, Liu CK. Clinical applications of susceptibility weighted imaging in patients with major stroke. J Neurol. 2012;259(7):1426-1432. doi:10.1007/s00415-0116369-2

26. Goos JD, van der Flier WM, Knol DL, et al. Clinical relevance of improved microbleed detection by susceptibility-weighted magnetic resonance imaging. Stroke. 2011;42(7):1894-1900. doi:10.1161/ STROKEAHA.110.599837
Neuropsychiatric Disease and Treatment

\section{Publish your work in this journal}

Neuropsychiatric Disease and Treatment is an international, peerreviewed journal of clinical therapeutics and pharmacology focusing on concise rapid reporting of clinical or pre-clinical studies on a range of neuropsychiatric and neurological disorders. This journal is indexed on PubMed Central, the 'PsycINFO' database and CAS, and is the official journal of The International Neuropsychiatric Association (INA). The manuscript management system is completely online and includes a very quick and fair peer-review system, which is all easy to use. Visit http://www.dovepress.com/testimonials.php to read real quotes from published authors. 